\title{
Inhibition of pancreatic cancer and potentiation of gemcitabine effects by the extract of Pao Pereira
}

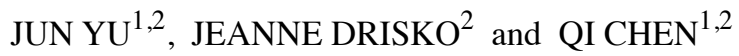 \\ ${ }^{1}$ Department of Pharmacology, Toxicology and Therapeutics, ${ }^{2}$ University of Kansas Integrative Medicine, \\ University of Kansas Medical Center, Kansas City, KS 66160, USA
}

Received January 25, 2013; Accepted March 19, 2013

DOI: $10.3892 /$ or.2013.2461

\begin{abstract}
Lack of effective therapy is a major problem in the treatment of pancreatic cancer. In the present study, we investigated a natural product, the extract of Pao Pereira (Pao), for its anti-pancreatic cancer effect in vitro and in vivo, either alone or in combination with the first-line chemotherapeutic drug gemcitabine (Gem). Pao induced dose-dependent apoptosis to all five tested pancreatic cancer cell lines. The combination of Pao and Gem had a synergistic effect in the inhibition of cell growth, with combination indices (CIs) $<1$ by Chou-Talalay's median effect analysis based on the isobologram principle. Adding Pao to Gem treatment reduced the concentration of Gem to produce an equitoxic effect on pancreatic cancer cells. In an orthotopic pancreatic xenograft mouse model, mice bearing PACN-1 tumors were treated with Pao and Gem, either alone or in combination. The progression of tumors was monitored longitudinally by imaging of live animals. While Gem did not provide significant inhibition, Pao treatment significantly suppressed tumor growth by $70-72 \%$. Combined Pao and Gem treatment further enhanced the tumor inhibitory effect compared to Gem alone, and markedly reduced metastatic lesions in the peritoneum. Collectively, these data suggest that the extract of Pao possesses anti-pancreatic cancer activity and can enhance the effects of Gem in vitro and in vivo.
\end{abstract}

Correspondence to: Professor Qi Chen, Department of Pharmacology, Toxicology and Therapeutics, University of Kansas Medical Center, 3901 Rainbow Boulevard MS1017, Kansas City, KS 66160, USA

E-mail: qchen@kumc.edu

Abbreviations: CI, combination index; Gem, gemcitabine; DRI, dosereduction index; H\&E, haematoxylin and eosin; i.p., intraperitoneal; MTT, 3-(4,5-dimethylthiazol-2-yl)-2,5-diphenyltetrazolium bromide; PARP, poly-ADP ribose polymerase; PI, propidium iodine; $\mathrm{Pao}, \mathrm{Pao}$ Pereira extract

Key words: Pao Pereira, plant extract, gemcitabine, synergy, pancreatic cancer

\section{Introduction}

Pancreatic cancer is one of the most lethal types of cancer worldwide and is the 4th leading cause of cancer-related mortality in the USA despite being responsible for only $2 \%$ of all new cancers diagnosed (1-3). Treatment effect and prognosis for pancreatic cancer remain dismal. Patients with this disease have an overall 5-year survival rate of only $3-5 \%$, which has remained essentially unchanged over the past 30 years (1-3). This is likely due to the limited treatment options, in addition to the aggressive nature of this disease, and the lack of early diagnostic tools. While surgical resection is the only potentially curative treatment for patients with pancreatic cancer, only $15-20 \%$ of patients have resectable disease at the time of diagnosis. Furthermore, patients who undergo surgery (Whipple procedure) have a perioperative mortality of 4-18\% and an additional risk of post operational complications (4-6). Nearly $100 \%$ of patients with pancreatic cancer develop metastases and succumb to the disease due to the debilitating metabolic effects of unrestrained tumor growth (6). Although there have been numerous attempts to develop improved systemic therapies of pancreatic cancer, gemcitabine (Gem) as a single agent remains the current standard of care (7). Gem as first-line therapy has a 12-month survival advantage compared with fluorouracil therapy (8). A new Gem-free regimen FOLFIRINOX combining 5-fluorouracil, leucovorin, irinotecan and oxaliplatin provided a 5-month survival benefit over Gem (9). However, this regimen added adverse effects. Lack of effective therapeutic options, lack of adjuvant therapy, significant side-effects with existing chemotherapies and radiation therapies or their combinations remain major problems in the treatment of pancreatic cancer.

Natural products have long been proven a considerable resource for bioactive anticancer agents. One of the advantages of natural products is their low toxicity compared with conventional chemo-drugs. Combination of natural compounds and standard chemotherapeutic drugs may exert additive or synergistic effects in killing cancer cells, which would in turn allow lower and safer doses to be used. Here, we investigated a plant extract for its activity against pancreatic cancer. Herbal preparation of Pao Pereira (Pao), a rainforest tree in the family of Apocynaceae, has long been used by oncologic patients and practitioners in complementary and alternative 
medicine. However, its anticancer activities against pancreatic cancer have yet to be systematically studied. A $\beta$-carboline alkaloid-enriched extract of Pao has recently been reported to suppress prostate cancer cells (10). Previous studies have shown the potential of $\beta$-carboline alkaloids against several tumors (11-13). In the present study, we investigated the activities of the $\beta$-carboline alkaloid-enriched extract of Pao (14) against pancreatic ductal adenocarcinoma, using multiple cell lines and a mouse model. The combination effect of the extract with Gem was also investigated.

\section{Materials and methods}

Cell lines and reagents. Human pancreatic cancer cell lines PANC-1, AsPC-1, HPAF-II, BxPC-3 and MiaPaCa-2 were obtained from the American Type Culture Collection (ATCC, Manassas, VA, USA). Immortalized human lung epithelial cells MRC-5 were provided by Dr Sittampalam at the University of Kansas Medical Center, and were used as a comparison to cancer cells. All the cells were cultured at $37^{\circ} \mathrm{C}$ in $5 \% \mathrm{CO}_{2} / 95 \%$ air in recommended growth media containing $10 \%$ fetal calf serum. Pao extract was provided by Natural Source International (New York, NY, USA). Pao and Gem (Sigma, St. Louis, MO, USA) were prepared in sterile water and stock at $-20^{\circ} \mathrm{C}$.

Cell viability assay. Cells were assessed for viability by 3-(4,5-dimethylthiazol-2-yl)-2,5-diphenyltetrazolium bromide (MTT) assay at $72 \mathrm{~h}$ of treatment. Cells in exponential growth phase were exposed to serial dilutions of Pao, Gem, or the combination of the two, for $72 \mathrm{~h}$. Then, cells were changed into fresh media containing MTT and were incubated for $4 \mathrm{~h}$. The colorimetric MTT assay assessed relative proliferation, based on the ability of living, but not dead cells, to reduce MTT to formazan $(15,16)$. Cells did not reach plateau phase during the incubation period. Fifty percent inhibitory concentration $\left(\mathrm{IC}_{50}\right)$ was defined as the concentration of drug that inhibited cell growth by $50 \%$ relative to the untreated control. Pilot experiments for each cell line were performed to optimize cell density and assay duration and to center drug dilution series approximately on the $\mathrm{IC}_{50}$.

Soft agar colony formation assay. Anchorage-independent colony formation assay in soft agar was utilized to determine survival of tumorigenic cancer cells following the treatments. In 6-well plates, PANC-1 cells $(5,000$ cells/well) were seeded in the upper layer containing $0.5 \%$ agar, DMEM medium, and $10 \%$ fetal bovine serum (FBS), with or without $400 \mu \mathrm{g} / \mathrm{ml} \mathrm{Pao.}$ The solid agar base (lower layer) contained $0.75 \%$ agar, DMEM medium, $10 \%$ FBS with or without $400 \mu \mathrm{g} / \mathrm{ml}$ Pao, respectively. The number of colonies was counted after 20 days.

Apoptosis detection by flow cytometry. Cells were exposed to various concentrations of Pao for $48 \mathrm{~h}$. Cells were washed in PBS, resuspended in binding buffer, and subjected to FITCconjugated Annexin V and propidium iodide (PI) staining according to the manufacturer's protocol (BD Biosciences, San Jose, CA, USA). Cells were analyzed by flow cytometry. Annexin $\mathrm{V}$ positive cells and Annexin V-PI double positive cells were identified as early and late stage apoptotic cells, respectively. PI single-positive cells were identified as necrotic cells.

Western blot analysis. A total of $40 \mu \mathrm{g}$ protein was loaded for SDS-polyacrylamide gel electrophoresis. Primary and secondary antibodies were from Cell Signaling Technology Inc. (Danvers, MA, USA): rabbit anti-poly-(ADP-ribose)polymerase (PARP) $(1: 2,000)$, rabbit anti-caspase-3 $(1: 1,000)$, rabbit anti-capase-8 $(1: 1,000)$, mouse anti- $\beta$-actin $(1: 1,000)$, and goat anti-rabbit or anti-mouse $\operatorname{IgG}(1: 5,000)$. Blots were developed using immobilon chemiluminescent substrate (Thermo Fisher Scientific, Waltham, MA, USA).

Intraperitoneal pancreatic cancer mouse model. Animal experiments were conducted following a protocol (\#20122035) approved by the Institutional Animal Care and Use Committee. Through an intra-pancreas surgical procedure, the human pancreatic ductal adenocarcinoma cells PANC-1 were orthotopically implanted into the pancreas of nude mice (3.2 $\left.\times 10^{5} / \mathrm{mice}\right)$. Ten days after tumor cells were implanted, treatment began with intraperitoneal (i.p.) injection of Gem (20 mg/kg, every 4 days), Pao (20 or $50 \mathrm{mg} / \mathrm{kg}$ daily), the respective combination of Gem and Pao and saline as control. To allow in vivo imaging, PANC-1 cells were transfected with luciferase gene. Luciferin at $150 \mathrm{mg} / \mathrm{kg}$ was administered i.p. each time prior to imaging for the luminance of tumor cells. Mice were kept anesthetized with $\sim 2.5 \%$ isoflurane. An IVIS in vivo imaging system (Caliper Life Sciences, Hopkinton, MA, USA) was used to scan the mice. The Living Image 4.1 software (Caliper Life Sciences) was used for analysis of the images. After 70 days of treatment, mice were euthanized. All tumor lesions in the peritoneal cavity were collected and weighed. Major organs such as liver, kidney and spleen were subjected to histological analysis for any damage due to potential drug toxicity.

Data analysis. MTT data were normalized to their corresponding untreated controls for each condition (drug and cell type) and were expressed as percentage viability. Dose-reduction index (DRI) values for Gem were calculated by the equation $\mathrm{DRI} \mathrm{ICx}_{\mathrm{ICx}}=\left(\mathrm{D}_{\mathrm{Gem}} / \mathrm{D}_{\mathrm{Gem}+\mathrm{Pao}}\right)$, where $\mathrm{D}_{\mathrm{Gem}}$ is the dose of Gem alone required to produce an ICx level of cytotoxicity, and the divisor $\mathrm{D}_{\mathrm{Gem}+\mathrm{Pao}}$ is the dose of Gem needed to produce the same ICx level of cytotoxicity when it is combined with Pao (at a given molar ratio). $\mathrm{DRI}_{\mathrm{Gem}}$ is defined with respect to Gem. Combination index $(\mathrm{CI})$ values were calculated by the equation $\mathrm{CI}_{\mathrm{ICx}}=\left(\mathrm{D}_{\mathrm{GemCombo} \mathrm{ICx}_{\mathrm{X}}} / \mathrm{D}_{\mathrm{Gem} \mathrm{ICx}}\right)$ $+\left(\mathrm{D}_{\text {PaoCombo ICX }} / \mathrm{D}_{\text {Pao ICX }}\right)+\alpha\left[\left(\mathrm{D}_{\text {GemCombolCx }}\right)\left(\mathrm{D}_{\text {PaoComboIC }}\right) /\left(\mathrm{D}_{\mathrm{Gem} \mathrm{ICX}}\right)\right.$ $\left.\left(D_{\text {Pao ICx }}\right)\right]$, where $\mathrm{D}$ is the dose of Gem and Pao either alone or in combination at a given constant ratio required to produce an ICx level of cytotoxicity (17-19). The more conservative assumption of mutual exclusivity was adopted $(\alpha=0)$. SPSS15.0 was used for additional statistical analysis.

\section{Results}

Effect of Pao extract against pancreatic cancer cells. Human pancreatic cancer cell lines (PANC-1, AsPC-1, HPAF-II, MiaPaCa-2 and BxPC-3) were compared to an immortalized epithelial non-tumorigenic cell line (MRC-5) for sensitivity to 
A

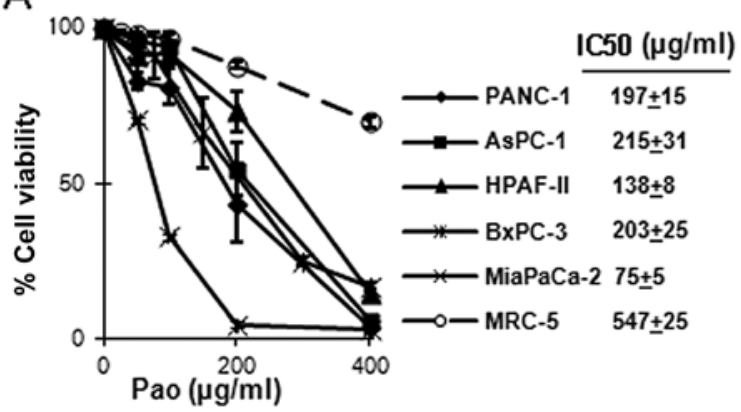

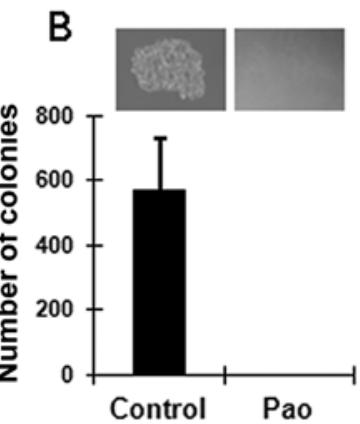

Figure 1. Cytotoxicity of Pao Pereira (Pao) in normal cells and pancreatic cancer cells. (A) Dose-response curves. Human pancreatic cancer cells PANC-1, AsPC-1, HPAF-II, BxPC-3 and MiaPaCa-2 were exposed to serial concentrations of Pao for 72 h. Cell viability was detected by MTT assay. An immortalized non-cancerous epithelial cell MCR-5 was subjected to the same treatment. $\mathrm{IC}_{50}$ was defined as the concentration of Pao that inhibited cell viability by $50 \%$ relative to the untreated control. (B) Colony formation of PANC-1 cells in soft agar with and without Pao treatment. Five thousand PANC-1 cells/well in 6-well plates were either treated with $400 \mu \mathrm{g} / \mathrm{ml}$ Pao or untreated (Control). All values are expressed as means \pm SD of 3 independent experiments.
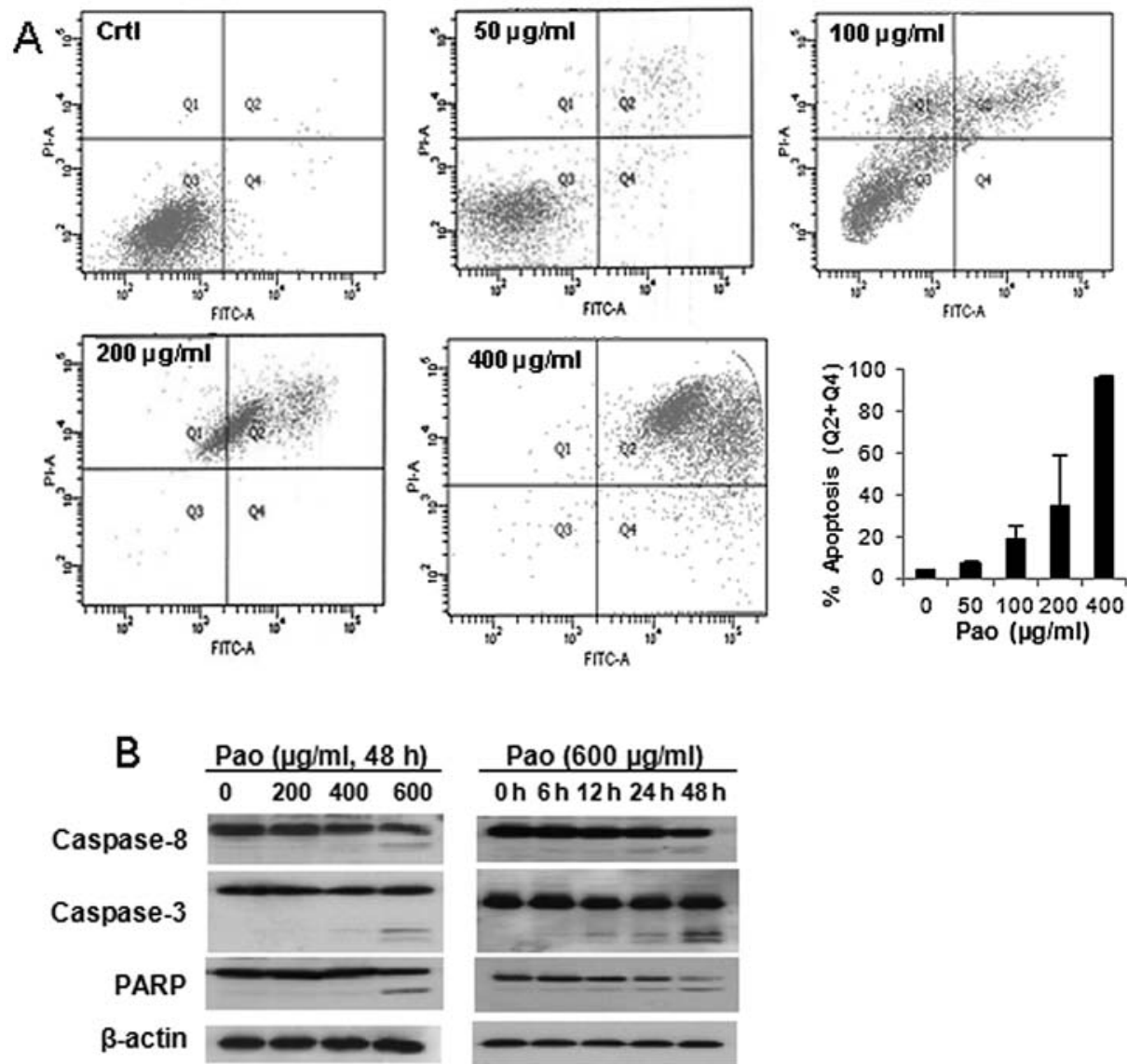

Figure 2. Apoptosis in PANC-1 cells induced by Pao Pereira (Pao). (A) Flow cytometry detection of apoptotic cells. PANC-1 cells were treated with Pao at the indicated concentrations. At $48 \mathrm{~h}$ of treatment, cells were subjected to FITC affiliated Annexin V and propidium iodine (PI) double staining and flow cytometry. Cells in Q2 and Q4 were identified as apoptotic cells, and cells in Q1 (PI positive only) were identified as necrotic cells. The percentage of apoptotic cells in each treatment was quantified and is shown in the bar graph. (B) Cleavage of caspase-8, caspase-3 and PARP in PANC-1 cells treated with Pao. Cells were treated with Pao at different concentrations (left panel) and for different times (right panel) as indicated. The cleavage of caspase-8, caspase-3 and PARP was detected by western blots.

Pao. All the cancer cells were susceptible to Pao treatment with $\mathrm{IC}_{50}$ ranging from 75 to $215 \mu \mathrm{g} / \mathrm{ml}$. MRC-5 cells were less sensitive to the same treatment with an $\mathrm{IC}_{50}$ of $547 \mu \mathrm{g} / \mathrm{ml}$ (Fig. 1A). At the concentration of $400 \mu \mathrm{g} / \mathrm{ml}$ Pao selectively killed $85-100 \%$ of cancer cells, and only decreased viability in MRC-5 cells for $25 \%$. Colony formation in soft agar was used to assess the survival of tumorigenic cancer cells, which has been positively correlated to in vivo tumorigenicity of the cancer cells in animal models $(20,21)$. Untreated PANC-1 cells formed colonies in soft agar at a rate of $12 \%$. Pao at $400 \mu \mathrm{g} / \mathrm{ml}$ completely inhibited formation of colonies of PANC-1 cells in soft agar (Fig. 1B), indicating no survival of tumorigenic cancer cells with this treatment. 


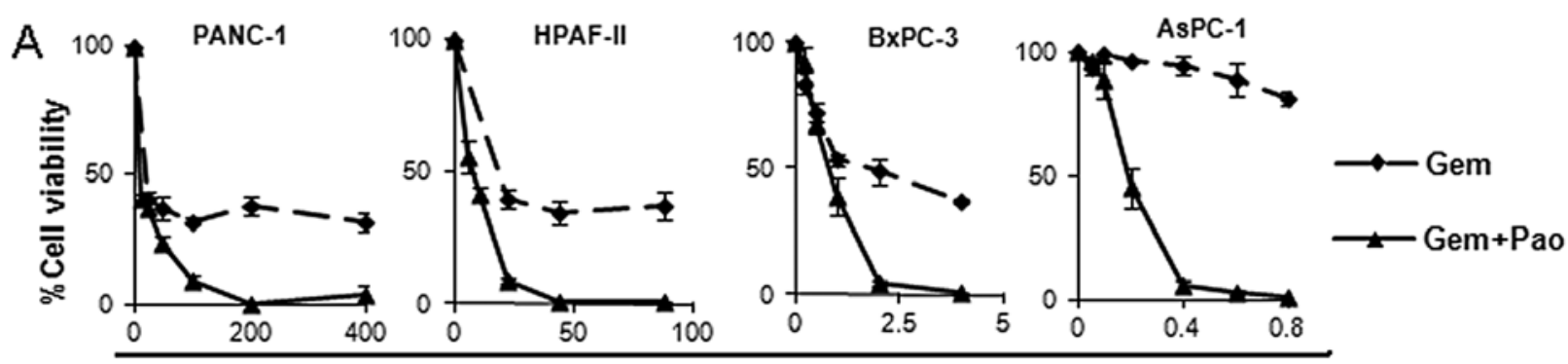

Gemcitabine $(\mu \mathrm{M})$
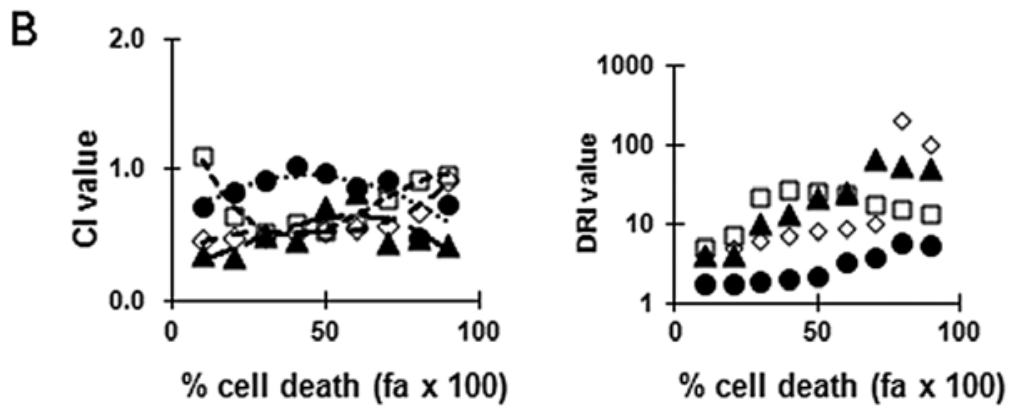

Figure 3. Combination effect of Pao Pereira (Pao) and gemcitabine (Gem) against pancreatic cancer cells. (A) Dose-response curves of pancreatic cancer cells to Gem, or Gem and Pao combination (Gem+Pao). Cells were treated with Gem (dotted line) and the combination of Gem and Pao (Gem+Pao, solid line) for $72 \mathrm{~h}$ to obtain optimum effect with Gem. The combination had the molar ratio of $\mathrm{IC}_{50 \mathrm{~Pa}}: \mathrm{IC}_{50 \mathrm{Gem}}$, and was plotted against Gem concentration. (B) Combination index (CI, left panel) across the fraction affected $\left(\mathrm{f}_{\mathrm{a}}\right) . \mathrm{CI}>1$ indicates antagonism, $\mathrm{CI}=1$ indicates additive effect, and $\mathrm{CI}<1$ indicates synergy. Dose-reduction index (DRI, right panel) across the $\mathrm{f}_{\mathrm{a}}$ for Gem when Pao was added.

To assess Pao-induced death pathway, Annexin V/PI staining was performed to detect apoptosis vs. necrosis in PANC-1 cells treated with Pao. Flow cytometry demonstrated that the percentage of cells positive with Annexin V/PI staining increased from $4.3 \%$ in untreated cells to the highest, $96 \%$, in $400 \mu \mathrm{g} / \mathrm{ml}$ Pao-treated cells (Fig. 2A). The induction of apoptosis was dependent on concentrations of Pao. Apoptosis contributed to the majority of cell death, while necrosis contributed to only $2-16.5 \%$ of cell death at all tested concentrations. As a consistent finding, cleavage of caspase-8, caspase- 3 and PARP was also detected in Pao-treated PANC-1 cells. The cleavage was induced dependent on the concentration of Pao and time of treatment (Fig. 2B).

Synergistic effect of Pao in combination with Gem against pancreatic cancer cells. After determining the doseresponse relationships to Pao in the cancer cells (Fig. 1A), the dose-response relationships to $\mathrm{Gem}$ were also established in PANC-1, AsPC-1, HPAF-II and BxPC-3 cells (Fig. 3A, dotted lines). A constant ratio design was used to systematically examine the combination effect of Gem and Pao $(\mathrm{Gem}+\mathrm{Pao})$. Ratio of Gem:Pao was chosen as $\mathrm{IC}_{50 \mathrm{Gem}}: \mathrm{IC}_{50 \mathrm{~Pa} a}$. Cell viability was examined after $72 \mathrm{~h}$ of treatment to obtain the optimum Gem effects. Results clearly showed that when Pao was added to Gem, drops in cell viability were markedly enhanced in all tested cells, compared to Gem as a single agent (Fig. 3A). The enhancement of the cytotoxic effect was particularly obvious in AsPC-1 cells which were more resistant to Gem treatment.

The combination index (CI) for each cell type was calculated based on the isobologram principle to examine whether drug combinations may be synergistic $(\mathrm{CI}<1)$, additive $(\mathrm{CI}=1)$, or antagonistic (CI>1) (17-19). In addition, DRI for Gem was calculated to indicate whether Gem concentration can be reduced when Pao was combined to produce the same cytotoxic effect. DRI values of $>1$ indicate a favorable combination. $\mathrm{DRI}<1$ is interpreted as an antagonistic combination. In all cell lines tested, CI values were $\leq 1$ and DRIs were $>1$ (Fig. 3B) across the desired levels of effect (fraction affected, $f_{a}$ ), indicating additive to synergistic effect when Pao was combined with Gem. These data also showed that the concentration of Gem is decreased to produce an equitoxic effect on pancreatic cancer cells when Pao is combined.

In vivo tumor inhibitory effect of Pao either alone or in combination with Gem. An orthotopic pancreatic cancer mouse model was used to evaluate the effect of Pao and Gem plus Pao $(\mathrm{Gem}+\mathrm{Pao})$ treatment. Compared to a subcutaneous xenograft model, this model better mimics clinical conditions of human pancreatic cancer, as the local environment for pancreatic cancer development was represented.

Treatment was carried out as described in Materials and methods. Tumor progress was monitored through longitudinal live animal imaging. Representative images in 5 mice from each group are shown in Fig. 4A. Gem at an early stage of the treatment showed tumor inhibitory effects (day 20, Fig. 4A), but it failed to inhibit the tumor at a later stage (day 69, Fig. 4A). At the end of experiment, Gem-treated mice had only insignificant reduction of tumor burden. By contrast, Pao at either $50 \mathrm{mg} / \mathrm{kg}$ or a lower dose of $20 \mathrm{mg} / \mathrm{kg}$ strongly inhibited tumor growth throughout the treatment period, and resulted in significantly reduced tumor burden at the end of experiment. 
A

Control

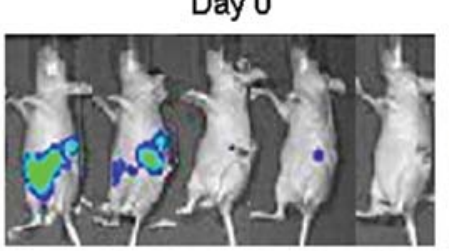

Pao20

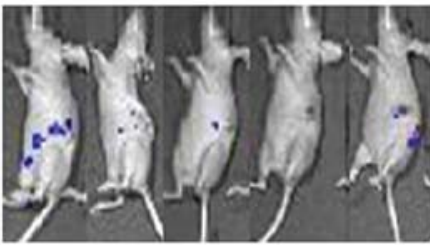

Pao50

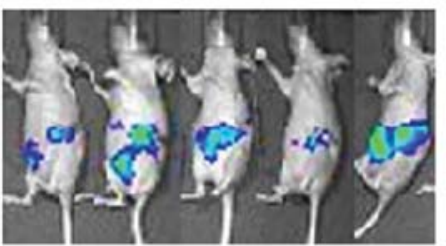

Gem

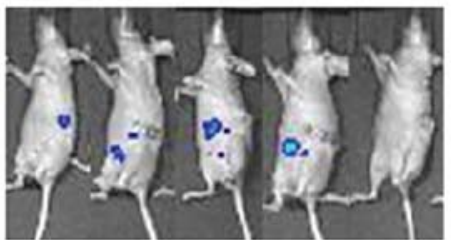

Gem+Pao20

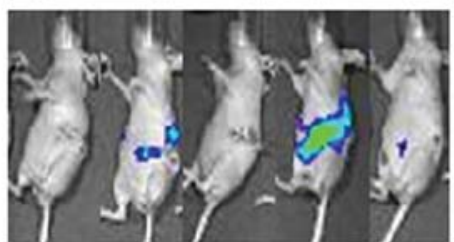

Gem+Pao50

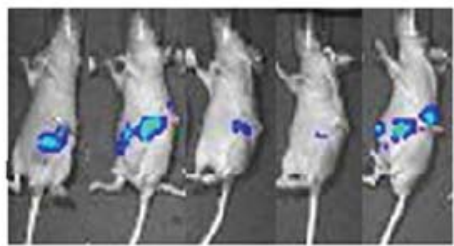

Day 20
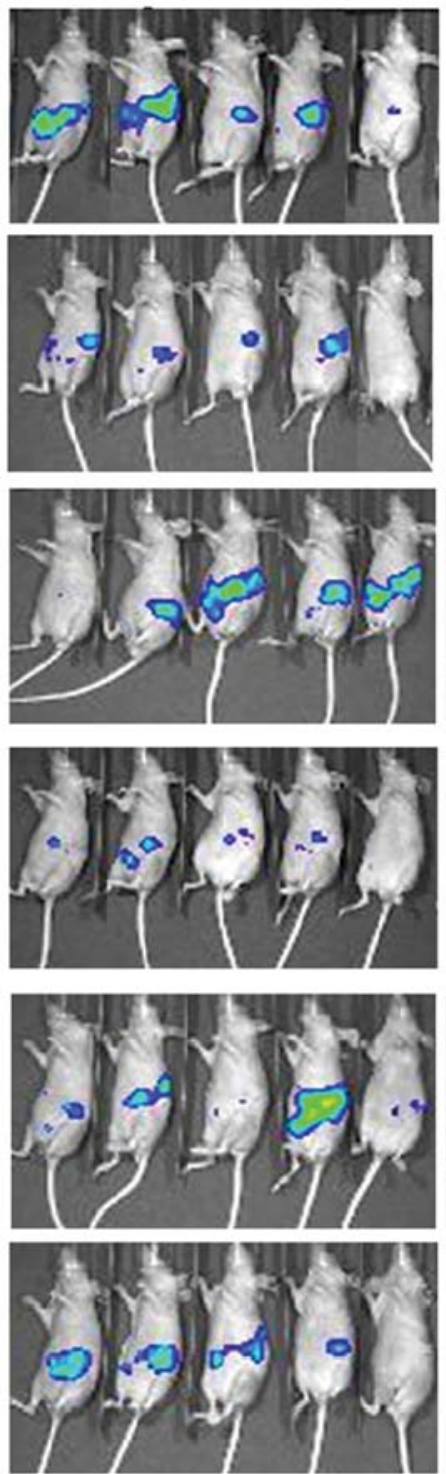

Day 69
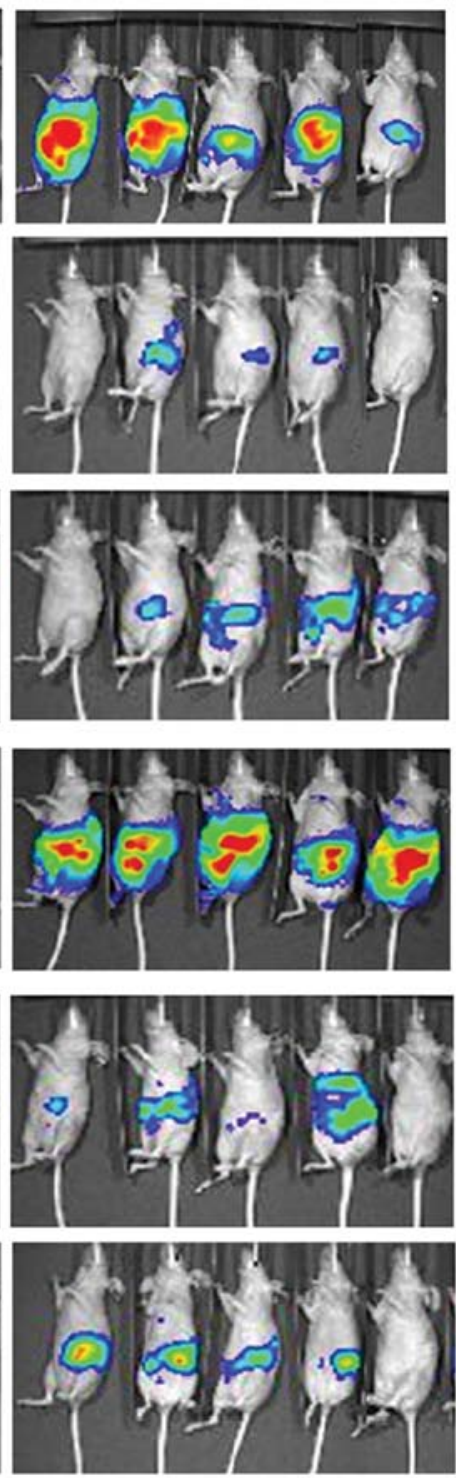

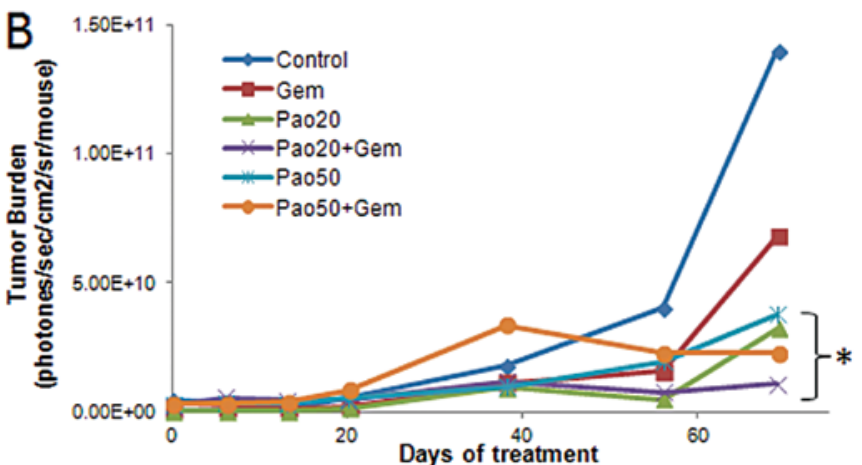

Of note, the combination treatments mimicked the effect of Pao treatment alone, without showing additional effects with added Gem. These observations were confirmed by quantification of bioluminescence intensity in all images to show total tumor burden (Fig. 4B). Gem did not provide significant inhibition of the tumor at the end of the treatment. By contrast, a significant inhibition was shown between the control group and the Pao-presenting treatment groups. There was no diffe-
Figure 4. Live animal imaging. PANC-1 cells were orthotopically implanted into the pancreas of nude mice $\left(3.2 \times 10^{5} / \mathrm{mice}\right)$. After 10 days of tumor inoculation, treatment began (day 0) with the doses indicated in Materials and methods. To allow for in vivo imaging, PANC-1 cells were transfected with luciferase gene, and $150 \mathrm{mg} / \mathrm{kg}$ Luciferin was administered i.p. each time before imaging for the luminance of tumor cells. (A) Representative images of 5 mice from each group at day 0 , day 20 and day 69. (B) Longitudinal tumor growth shown by quantification of all images in each treatment group. Tumor burden is represented by average total photon flux in each mouse. $\mathrm{n}=7$ for control; $\mathrm{n}=9$ for Gem, $\mathrm{n}=9$ for Pao20; $\mathrm{n}=10$ for Gem+Pao20; $\mathrm{n}=8$ for Pao50; and $\mathrm{n}=10$ for Gem+Pao50. ${ }^{*} \mathrm{P}<0.05$ vs. control group. Pao, Pao Pereira; Gem, gemcitabine.

rence whether Gem was present or absent in the Pao-treated groups. Both low $(20 \mathrm{mg} / \mathrm{kg})$ and high dose $(50 \mathrm{mg} / \mathrm{kg})$ of Pao provided similar effects. A dose-dependent effect to Pao was absent at the tested doses.

At necropsy, all tumors were weighed and metastatic lesions were counted. The results clearly confirmed data from live animal imaging (Fig. 5). Whereas Gem had minimal reduction on total tumor weight $(\mathrm{P}=0.25)$, Pao alone decreased tumor 
A

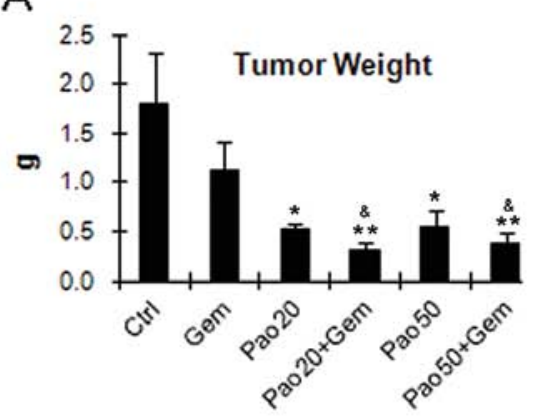

B

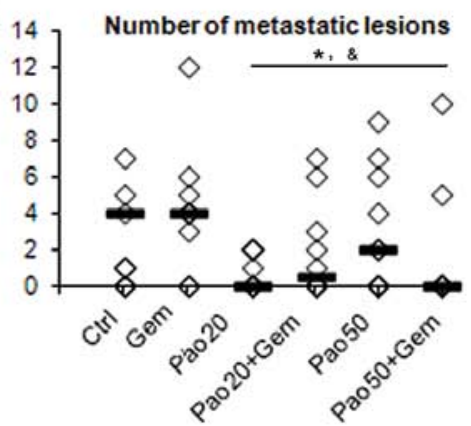

C

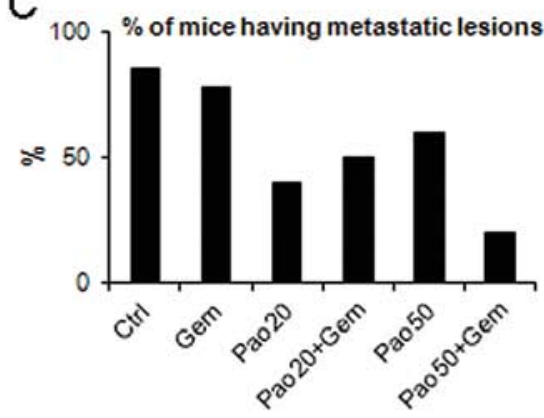

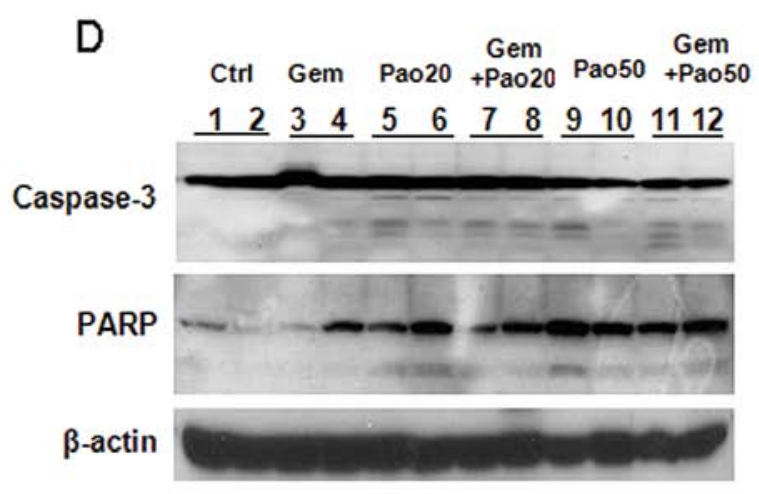

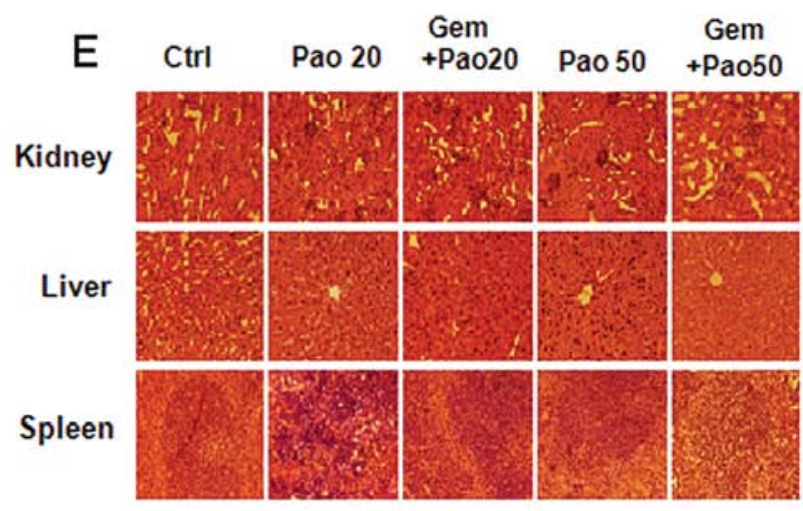

Figure 5. Inhibition of tumor weight and metastasis by Pao Pereira (Pao) and the combination of Pao and gemcitabine (Gem) in vivo. Mice bearing orthotopic PANC-1 tumors were treated for 70 days. Tumor weight and metastasis were assessed at the end of the treatment by necropsy. (A) Total tumor weight. (B) Number of metastatic lesions in each mouse. The bars show median number of metastatic lesions in each group. (C) Percentages of mice with metastasis. $\mathrm{n}=7$ for control; $\mathrm{n}=9$ for Gem, $\mathrm{n}=9$ for Pao20; $\mathrm{n}=10$ for $\mathrm{Gem}+\mathrm{Pao} 20 ; \mathrm{n}=8$ for Pao50; and $\mathrm{n}=10$ for Gem+Pao50. ${ }^{*} \mathrm{P}<0.05$, ${ }^{* *} \mathrm{P}<0.01$ vs. control group; ${ }^{\&} \mathrm{P}<0.05$ vs. Gem group. (D) Cleavage of caspase-3 and PARP in tumor samples from different treatment groups. Tumors of 2 mice from each group were examined. (E) H\&E staining of major organs from different treatment groups (original magnification, x400). Kidney, liver and spleen were collected from each group and fixed in $4 \%$ formaldehyde, and were later subjected to histological analysis.

weight by $72 \%$ at $20 \mathrm{mg} / \mathrm{kg} / \mathrm{day}$ and by $70 \%$ at $50 \mathrm{mg} / \mathrm{kg} / \mathrm{day}$ $(\mathrm{P}<0.05$ for both groups vs. control) (Fig. 5A). The Pao-treated groups had significantly lighter tumor burden compared to the Gem-treated group $(\mathrm{P}<0.05)$. By combining Pao and Gem, tumor weight decreased by $82 \%(\mathrm{Gem}+20 \mathrm{mg} / \mathrm{kg}$ Pao) and by $78 \%(\mathrm{Gem}+50 \mathrm{mg} / \mathrm{kg}$ Pao $)$ relative to control. Notably, the enhancement was not significant compared to Pao single-drug treatment, but was highly significant compared to Gem singledrug treatment. Again, there was no dose-dependence to Pao, consistent with imaging.

Regarding metastasis, the effect mirrored what was described above. Gem did not inhibit the formation of metastatic lesions in mice. Pao at both tested doses markedly inhibited the number of metastatic lesions (Fig. 5B). Pao treatment also decreased the number of mice that developed metastasis disease (Fig. 5C), while Gem failed to do so. The combinations of Gem+Pao had better effect compared to Gem in reducing either number of mice having metastasis, or the number of metastatic lesions in the mice, but had no difference compared to Pao treatment alone. There was no difference between the different doses of Pao.

Proteins were isolated from tumor samples of the treated and control mice. Western blot analysis showed cleavage of caspase- 3 and PARP in Pao and Gem+Pao treatment groups at all doses (Fig. 5D). These results confirmed the in vitro data that Pao induced apoptosis in tumor cells.
Pao treatment possessed low toxicity as no toxic effect was observed associated with the treatments. At the end of the experiments, major organs (kidney, liver and spleen) were subjected to haematoxylin and eosin (H\&E) staining and histological analysis. No tissue damage was detected in any of the groups (Fig. 5E).

\section{Discussion}

Gemcitabine (Gem) as the first-line therapy of pancreatic cancer provides little impact on the median survival for patients with locally advanced or metastatic pancreatic cancer $(22,23)$. Our study consistently observed that Gem only produced tumor inhibitory effects at early times of the treatment course when the tumors were small, but it failed to provide inhibition in tumor burden or metastasis in a longer time in our animal model. By contrast, the extract of Pao Pereira (Pao) exhibited strong inhibition in PANC-1 tumors throughout the course of the experiment, reaching $>70 \%$ inhibition even when tumors did not respond to Gem anymore. Consistent with the in vitro dose reduction effect for Gem, the combination of Pao and Gem had a better effect than Gem in vivo. However, the combination did not make a difference compared to Pao treatment alone in vivo. This is likely due to the fact that tumors did not respond to Gem treatment at a later course of the in vivo experiment. The Gem non-responsive tumor still 
responded to Pao treatment. These results greatly raise the potential for using $\mathrm{Pao}$ in pancreatic cancer treatment, alone or in addition to Gem.

Cell death and apoptosis induction showed dose-dependence on Pao concentrations in vitro, but such dose-response was lacking in the in vivo tumor inhibitory effects. The low $(20 \mathrm{mg} / \mathrm{kg})$ and high $(50 \mathrm{mg} / \mathrm{kg})$ doses of Pao had similar tumor inhibition in mice bearing PANC-1 tumors. Mice treated with $20 \mathrm{mg} / \mathrm{kg}$ Pao even had the tendency of fewer metastatic lesions than mice treated with $50 \mathrm{mg} / \mathrm{kg}$ Pao. In another study using Pao in prostate cancer, a similar doseindependence was observed (10). The reason for this treatment effect remains unknown. Since Pao was administered through intraperitoneal injection in our study, this effect was not likely due to the gastrointestinal absorption of bioactive components. However, it is possible that high concentrations of Pao could induce metabolic enzymes in vivo, potentially altering the chemical profile of the bioactive components to an inactive state. The effects of Pao in mice are probably a composite of multiple organ systems, unlike cells in culture systems. It is also possible that there is a threshold in the concentration that Pao executes antitumor effects in vivo, and above the threshold the effect might not be concentration-dependent, similar to the situation of the concentration-independent antibiotics vancomycin (24). Moreover, a U-shaped dose-response relationship was proposed to exist between essential nutrients and their biologic impact (25). Several studies have reported that a nonlinear relationship also applies to anticancer agents (26-29), although the mechanisms/reasons causing this effect remain unclear. Our data using the Pao extract appear to validate this hypothesis.

In addition to the substantial inhibitory effect against pancreatic cancer cells, Pao as a natural product had relatively low toxicity towards normal cells. Low toxicity was evident in mice treated with Pao where major organ toxicities were absent. In addition, by the dose-reduction effect, Pao allowed for lower concentrations of Gem while achieving an equivalent cytotoxicity in cancer cells with higher Gem concentrations alone. This may decrease the toxicity associated with chemotherapy.

Although the potential benefits of Pao were suggested in our study, the mechanism(s) underlying the Pao-induced anticancer effects warrant further study. A previous study reported DNA damage and cell cycle inhibition by Pao in prostate cancer cells (10). Our study showed that Pao mainly induced apoptosis both in vitro and in vivo as shown by flow cytometry and the cleavage of caspase-8, caspase-3 and PARP, which can be inter-connected to DNA damage and cell cycle inhibition. However, as this plant preparation contains a complex mixture of natural compounds, there is potential that it impacts multiple molecular targets and pathways that lead to apoptosis.

Preponderance of bioactive compounds is often found in medicinal plant and herbal mixtures, making them a notable source for discovery of novel drug leads. The $\beta$-carbolineenriched Pao extract could contain compounds that possess potent anticancer activity. Data presented here is the initial step in identifying the anticancer activity of Pao. Active components could be isolated and developed for optimizing efficacy, toxicity and other profiles that could lead to anticancer drug development.
Collectively, our data demonstrate the anti-pancreatic cancer activity of the Pao Pereira extract in vitro and in vivo. Further investigation is required for the use of this plant extract in the treatment of pancreatic cancer.

\section{Acknowledgements}

This study was supported by a grant from the Beljanski Foundation.

\section{References}

1. Lowenfels $\mathrm{AB}$ and Maisonneuve P: Epidemiology and prevention of pancreatic cancer. Jpn J Clin Oncol 34: 238-244, 2004.

2. Bayraktar S and Rocha-Lima CM: Advanced or metastatic pancreatic cancer: molecular targeted therapies. Mt Sinai J Med 77: 606-619, 2010.

3. ACS: Cancer Facts and Figures 2012. Available at: http://www. cancer.org/acs/groups/content/@epidemiologysurveilance/ documents/document/acspc-031941.pdf. (Accessed Jan 18, 2012).

4. Freelove R and Walling AD: Pancreatic cancer: diagnosis and management. Am Fam Physician 73: 485-492, 2006.

5. Hezel AF, Kimmelman AC, Stanger BZ, Bardeesy N and Depinho RA: Genetics and biology of pancreatic ductal adenocarcinoma. Genes Dev 20: 1218-1249, 2006.

6. Li D, Xie K, Wolff R and Abbruzzese JL: Pancreatic cancer. Lancet 363: 1049-1057, 2004

7. Cartwright T, Richards D and Boehm K: Cancer of the pancreas: are we making progress? a review of studies in the US Oncology Research Network. Cancer Control 15: 308-313, 2008.

8. Burris HA III, Moore MJ, Andersen J, Green MR, Rothenberg ML, Modiano MR, Cripps MC, Portenoy RK, Storniolo AM, Tarassoff P, Nelson R, Dorr FA, Stephens CD and Von Hoff DD: Improvements in survival and clinical benefit with gemcitabine as first-line therapy for patients with advanced pancreas cancer: a randomized trial. J Clin Oncol 15: 2403, 1997.

9. Conroy T, Desseigne F, Ychou M, Bouché O, Guimbaud R, Bécouarn Y, Adenis A, Raoul JL, Gourgou-Bourgade S, de la Fouchardière $\mathrm{C}$, Bennouna $\mathrm{J}$, Bachet JB, KhemissaAkouz F, Péré-Vergé D, Delbaldo C, Assenat E, Chauffert B, Michel P, Montoto-Grillot C and Ducreux M; Groupe Tumeurs Digestives of Unicancer; PRODIGE Intergroup: FOLFIRINOX versus gemcitabine for metastatic pancreatic cancer. N Engl J Med 364: 1817-1825, 2011.

10. Bemis DL, Capodice JL, Desai M, Katz AE and Buttyan R: beta-carboline alkaloid-enriched extract from the amazonian rain forest tree pao pereira suppresses prostate cancer cells. J Soc Integr Oncol 7: 59-65, 2009.

11. Beljanski M and Beljanski MS: Three alkaloids as selective destroyers of cancer cells in mice. Synergy with classic anticancer drugs. Oncology 43: 198-203, 1986.

12. Cao R, Chen Q, Hou X, Chen H, Guan H, Ma Y, Peng W and $\mathrm{Xu}$ A: Synthesis, acute toxicities, and antitumor effects of novel 9-substituted beta-carboline derivatives. Bioorg Med Chem 12: 4613-4623, 2004.

13. Chen Q, Chao R, Chen H, Hou X, Yan H, Zhou S, Peng W and $\mathrm{Xu}$ A: Antitumor and neurotoxic effects of novel harmine derivatives and structure-activity relationship analysis. Int J Cancer 114: 675-682, 2005.

14. Puiseux F, Le Hir A, Goutarel R, Janot MM and Lemen J: On the alkaloids of 'pao-pereira', Geissospermum laeve (Vellozo) Baillon. Note III. Geissoschizoline, apogeissoschizine and geissospermine. Ann Pharm Fr 17: 626-633, 1959 (In French).

15. Cole SP: Rapid chemosensitivity testing of human lung tumor cells using the MTT assay. Cancer Chemother Pharmacol 17: 259-263, 1986.

16. Denizot F and Lang R: Rapid colorimetric assay for cell growth and survival. Modifications to the tetrazolium dye procedure giving improved sensitivity and reliability. J Immunol Methods 89: 271-277, 1986.

17. Chou T and Talalay P: Applications of the median-effect principle for the assessment of low-dose risk of carcinogens and for the quantitation of synergism and antagonism of chemotherapeutic agents. In: New Avenues in Developmental Cancer Chemotherapy. Vol 8. Harrap KR and Connors TA (eds). Academic Press, New York, NY, pp37-64, 1987. 
18. Chou T, Rideout D, Chou J and Bertino J: Chemotherapeutic synergism, potentiation and antagonism. In: Encyclopedia of Human Biology. Vol 2. Dulbecco R (ed). Academic Press, Inc., San Diego, CA, pp371-379, 1991.

19. Chou TC: Theoretical basis, experimental design, and computerized simulation of synergism and antagonism in drug combination studies. Pharmacol Rev 58: 621-681, 2006.

20. Eagle H, Foley GE, Koprowski H, Lazarus H, Levine EM and Adams RA: Growth characteristics of virus-transformed cells. Maximum population density, inhibition by normal cells, serum requirement, growth in soft agar, and xenogeneic transplantability. J Exp Med 131: 863-879, 1970

21. Zeng G, Cai S, Liu Y and Wu GJ: METCAM/MUC18 augments migration, invasion, and tumorigenicity of human breast cancer SK-BR-3 cells. Gene 492: 229-238, 2012.

22. Oettle H, Post S, Neuhaus P, Gellert K, Langrehr J, Ridwelski K, Schramm H, Fahlke J, Zuelke C, Burkart C, Gutberlet K, Kettner E, Schmalenberg H, Weigang-Koehler K, Bechstein WO, Niedergethmann M, Schmidt-Wolf I, Roll L, Doerken B and Riess H: Adjuvant chemotherapy with gemcitabine vs observation in patients undergoing curative-intent resection of pancreatic cancer: a randomized controlled trial. JAMA 297: 267-277, 2007.

23. Renouf D and Moore M: Evolution of systemic therapy for advanced pancreatic cancer. Expert Rev Anticancer Ther 10: 529-540, 2010
24. Rybak MJ: The pharmacokinetic and pharmacodynamic properties of vancomycin. Clin Infect Dis 42 (Suppl 1): S35-S39, 2006.

25. Mertz W: The essential trace elements. Science 213: 1332-1338, 1981.

26. Nyberg F, Hou SM, Pershagen G and Lambert B: Dietary fruit and vegetables protect against somatic mutation in vivo, but low or high intake of carotenoids does not. Carcinogenesis 24: 689-696, 2003.

27. Donate F, Parry GC, Shaked Y, Hensley H, Guan X, Beck I, Tel-Tsur Z, Plunkett ML, Manuia M, Shaw DE, Kerbel RS and Mazar AP: Pharmacology of the novel antiangiogenic peptide ATN-161 (Ac-PHSCN-NH2): observation of a U-shaped doseresponse curve in several preclinical models of angiogenesis and tumor growth. Clin Cancer Res 14: 2137-2144, 2008.

28. Celik I, Surucu O, Dietz C, Heymach JV, Force J, Hoschele I, Becker CM, Folkman J and Kisker O: Therapeutic efficacy of endostatin exhibits a biphasic dose-response curve. Cancer Res 65: 11044-11050, 2005.

29. Nishi M, Ohba S, Hirata K and Miyake H: Dose-response relationship between coffee and the risk of pancreas cancer. Jpn J Clin Oncol 26: 42-48, 1996. 